\title{
A new knowledge synthesis method that is applicable to public policies
}

\section{F. Morestin}

The National Collaborating Centre for Healthy Public Policy (NCCHPP) has developed a method for synthesizing knowledge about public policies. This may be of interest to public health actors tackling chronic disease who are expected to provide comprehensive and contextualized evidence in order to inform policy making.

Some of the array of causes of chronic diseases have to be addressed through public policies. In this context, public health actors are called upon to produce knowledge syntheses in order to inform policy makers. But studying public policies raises specific challenges. Drawing inspiration from literature on the concept of evidence in public health, criteria involved in policy making, policy evaluation, deliberative processes as a means of collecting contextual knowledge, and methods proposed by established knowledge synthesis groups (such as the Community Preventive Services Task Force affiliated with the Centers for Disease Control and Prevention in the United States, the Cochrane Public Health Group in Australia, the National Institute for Health and Clinical Excellence in the United Kingdom), the NCCHPP has developed a method that is applicable to public policies.

This method guides rigorous knowledge syntheses on six dimensions relevant to the study of public policies (Figure 1): their effectiveness at preventing disease, their unintended effects, their equity-related issues (distribution of effects on different population groups), and their costs,
FIGURE 1

Relationships between the six dimensions for analyzing public policies

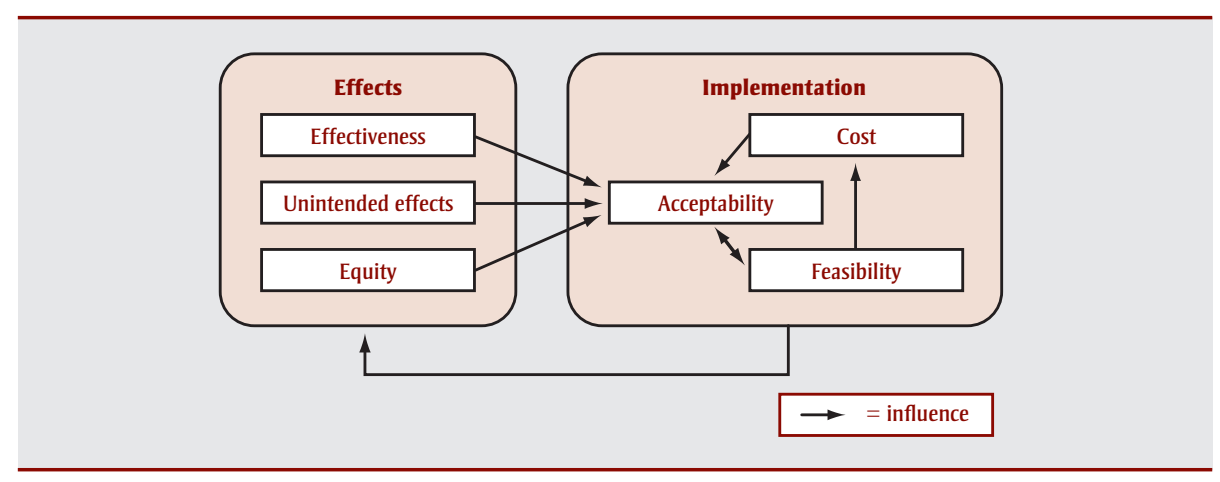

feasibility and acceptability-three kinds of implementation issues of concern to policy makers.

In order to gather knowledge on these different aspects in the most comprehensive and contextually relevant ways, the proposed process involves constructing the logic model of the policy under study, reviewing the scientific and grey literatures,

and organizing deliberative processes that bring together relevant stakeholders to gather contextual information regarding the potential local implementation of this policy (Figure 2).

The NCCHPP has produced a guide that presents the method step-by-step and incorporates questions to ask oneself, practical advice, and several tools for

FIGURE 2

Steps in the knowledge synthesis process

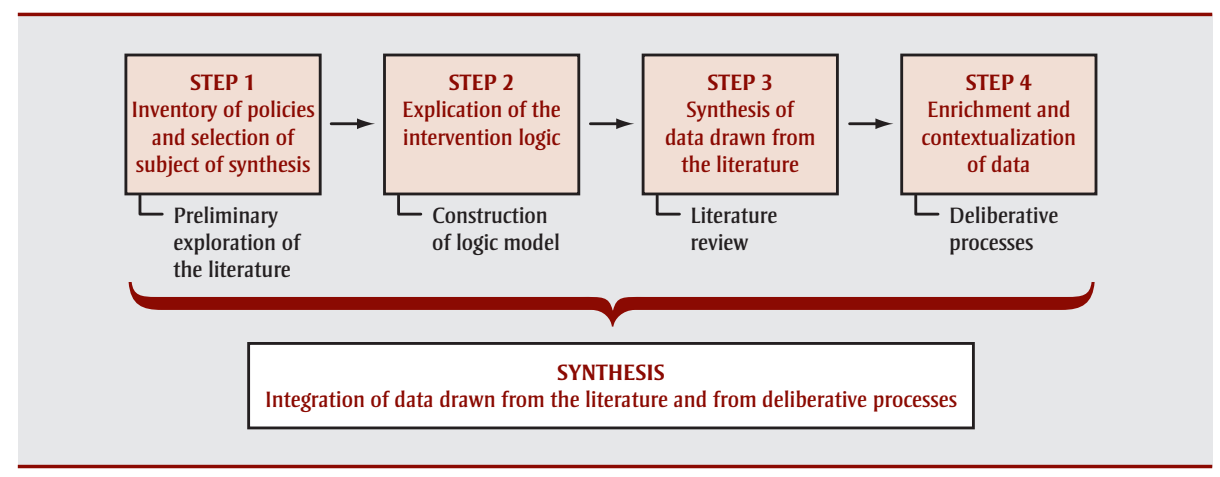


facilitating data collection and synthesis. The NCCHPP has also published a synthesis produced using this method, Public Policies on Nutrition Labelling: Effects and Implementation Issues - A Knowledge Synthesis; this document provides a concrete overview of how to use the proposed method and the kind of results that it can produce.

These documents are available in English and in French on the NCCHPP's website: http://www.ncchpp.ca/ 172/Publications.ccnpps. The NCCHPP also offers training material, workshops and methodological support to those who are interested in using this method. 